\title{
Psoriatic spondyloarthritis and Sjögren syndrome: a casual association?
}

\author{
V. Bruzzese ${ }^{1}$, C. Marrese ${ }^{1}$, P. Scolieri', J. Pepe ${ }^{2}$ \\ IInternal Medicine and Rheumatology Unit, P.O. S. Spirito-Nuovo Regina Margherita Hospital, Rome, Italy; \\ 2Department of Clinical, Internal, Anesthesiology and Cardiovascular Sciences, La Sapienza, \\ University of Rome, Italy
}

\section{SUMMARY}

The association between Sjögren syndrome (SS) and psoriatic arthritis (PsA) is rare. Herein, we report a case of SS in a PsA patient with the mutilans variant. A 67-year old woman developed PsA with progressive articular destruction up to the typical deformation of 'telescoping fingers' in the distal phalanges. Psoriatic onychopathy presented ten years after the osteolytic damage in the hands. This late appearance led to delayed diagnosis and therapy, and, consequently, worsened the articular destruction. Thereafter, the patient developed a typical SS with clinical symptoms, such as xerophthalmia and xerostomia. This diagnosis was confirmed by positive diagnostic tests, such as Schirmer test, ANA, and anti-SSA/Ro and anti-SSB/La antibodies. A potential association between the two diseases is discussed.

Key words: Psoriatic arthritis mutilans; Sjögren's syndrome; antinuclear antibodies; anti TNF-therapy.

Reumatismo, 2020; 72 (3): 170-172

\section{INTRODUCTION}

1 soriatic spondylarthritis (PsA) and Sjögren syndrome (SS) are two chronic inflammatory diseases, with different clinical and pathogenic characteristics. SS can be primitive or secondary ( $\mathrm{SSS}$ ) to other autoimmune diseases. In particular, it has been associated with rheumatoid arthritis (RA) and systemic lupus erythematosus (SLE) (1). In these cases, it remains unclear whether SS is an independent disease or part of the spectrum of symptoms of the primary disease. PsA is included in the large family of the so-called seronegative spondyloarthritides. Joint involvement may be peripheral or axial, and includes arthritis mutilans (AM) and distal interphalangeal arthritis (2). The onset of sSS during PsA is a very rare event, of which we describe a case.

Corresponding author: Jessica Pepe Department of Clinical, Internal, Anesthesiology and Cardiovascular

Sciences, La Sapienza

University of Rome, Italy

E-mail: jessica.pepe@uniroma1.it

\section{CASE REPORT}

A 67-year old woman with no family history of psoriasis or rheumatic diseases reported to have suffered from episodic ar- thralgia of the fingers since 15 years. She had no other diseases and the only drugs she had taken were non-steroidal anti-inflammatory drugs (NSAIDs) to self-treat her arthralgia. At the age of 55, osteolytic damage appeared in the distal phalanges of the $2^{\text {nd }}$ finger of the right hand and the $4^{\text {th }}$ finger of the left hand, with progressive articular destruction up to the typical deformity of telescoping fingers, thus leading to the diagnosis of PsA of the mutilans variant (Figures 1 and 2). Therapy with methotrexate $(10 \mathrm{mg} / \mathrm{wk})$, methylprednisolone (8 $\mathrm{mg}$ /daily) and NSAIDs was started without achieving any improvement. Moreover, the patient developed xerophthalmia and xerostomia, and a Schirmer's test resulted positive. Therefore, a biological therapy with etanercept ( $25 \mathrm{mg} \mathrm{x} 2 /$ wk) was initiated and determined a rapid improvement of all symptoms. However, due to her recurrent herpes simplex labialis infection, the patient decided to stop this therapy 2 years later. Subsequently, she had a joint pain relapse with the appearance of dactylitis in the fingers of the right hand.

At age of 62 years, the patient was seen by 
us for the first time. Due to her symptoms, in particular her eye involvement, a biochemical evaluation was performed. Laboratory blood tests revealed elevated ESR and CRP, positive antinuclear antibodies (1:640), anti ENA antibodies, including anti-SSA/Ro and anti-SSB/La antibodies. Anti nDNA antibodies, rheumatoid factor, and anti-cyclic citrullinated peptide antibodies were negative. Schirmer's test was positive ( $<5 \mathrm{~mm} / 5 \mathrm{~min})$. Moreover, severe destructive bone lesions of the fingers with typical telescoping fingers were documented. No signs of cutaneous psoriasis or psoriatic onychopathy were observed. Treatment with methotrexate $(10 \mathrm{mg} / \mathrm{wk})$ and adalimumab (40 mg/bi-monthly) was started and led to a good control of the disease. After 2 years, the patient discontinued again her therapy, and failed to undergo the scheduled clinical controls. Only in October 2017, she returned for a visit due to severe pain in her fingers and toes, xerostomia, and xerophthalmia. Psoriatic onychopathy was observed for the first time. Adalimumab therapy was re-started.

\section{DISCUSSION AND CONCLUSIONS}

Arthritis mutilans (AM) is part of the clinical manifestations of PsA, with a prevalence of $5 \%$ (3). It is characterized by progressive joint erosion and destruction of the phalanges of fingers and toes. AM generally occurs many years after the onset of PsA, although cases of early onset have been reported (4). Primary SS is a multisystem autoimmune disease involving exocrine glands, characterized by specific symptoms, such as xerophthalmia and xerostomia. SS may also develop during autoimmune rheumatic diseases as secondary form (sSS). In a recent systematic review, the prevalence of sSS was $19.5 \%$ in RA and $14 \%$ in SLE patients (5). An association between SS and ankylosing spondylitis was also described, with a prevalence of $10 \%$ (6). On the contrary, the association of PsA and SS has been rarely described and, according to our knowledge, a total of 18 probable cases have been reported (7-12).
While the onset of sSS, in either RA and SLE, is understandable from a pathogenic point of view, its appearance during the course of PsA is less clear. Indeed, PsA and SS are two different diseases with the autoinflammatory component being prevalent in the former and the autoimmune process in the latter. However, PsA may induce autoimmunity, as pointed out by frequent se-

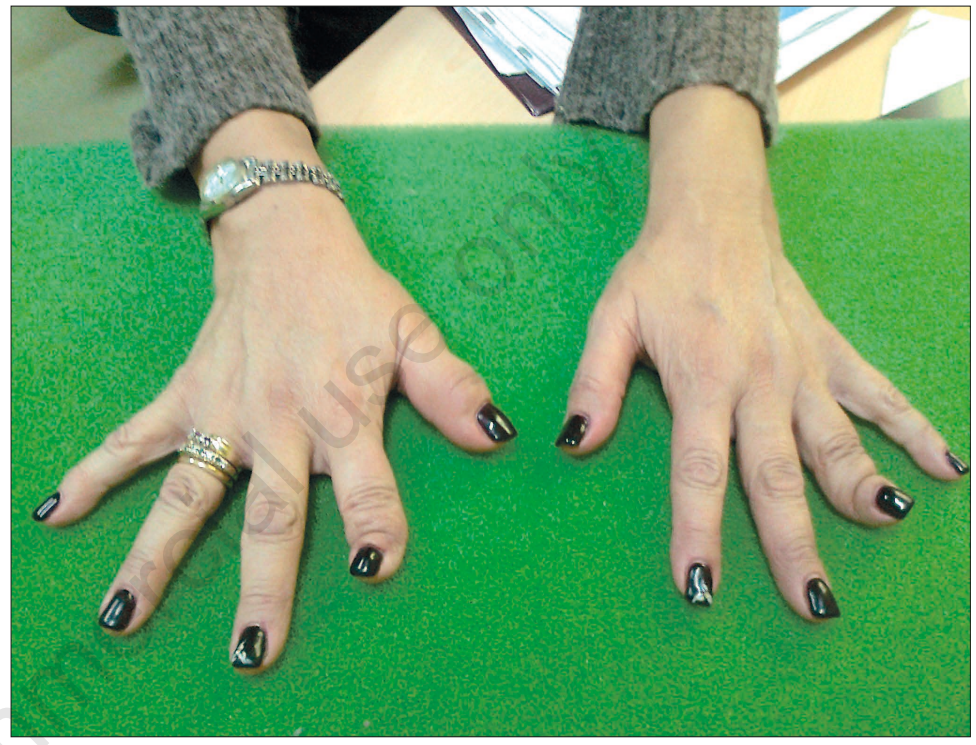

Figure 1 - Patient's hands with typical deformities at the distal phalanges of the $2^{\text {nd }}$ finger of the right hand and the $4^{\text {th }}$ finger of the left hand.

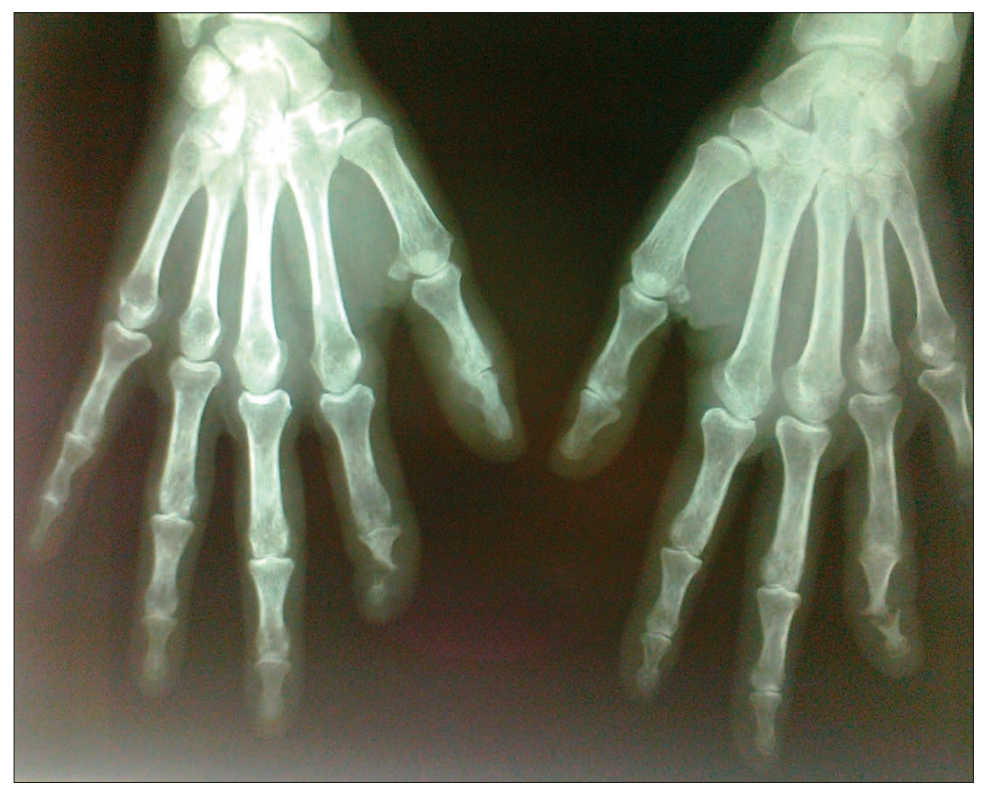

Figure 2 - Hands radiographs of the patient. 
ropositivity for antinuclear antibodies, and when this occurs, a concomitant SS is often present (8).

Besides the association between PsA and SS, our case presents some interesting peculiarities. The first unusual characteristic is the onset of AM in a subject without any familiarity or personal history of psoriasis accompanied by an accelerated evolution, even in the absence of cutaneous lesions. The second peculiarity is the development of a psoriatic onychopathy more than 10 years after the onset of erosive joint lesions. Nowadays, potential evidence of nail profile alterations can be detected with magnetic resonance imaging in psoriatic arthritis patients in the absence of clinically obvious nail lesions, but our patient did not perform this examination (13). Third, the diagnostic delay and the poor therapeutic adherence of the patient certainly worsened the destructive joint injuries leading to disability. In previous series, we found that the delay or lack of biologic therapy contributed remarkably to the evolution of AM (14). Lack of adherence to therapy in patients with PsA is one of the major causes that prevents from achievement of remission or minimal disease activity (15). Psychic conditions, such as stress, anxiety, depression and alexithymia are comorbidities that are often present in patients with PsA and lead to poor collaboration with the doctor and non-acceptance of the disease. For this reason, a multidisciplinary approach to PsA patients is recommended, also requiring the presence of a psychologist (16).

In conclusion, our case points to the importance of searching for signs and symptoms of SS in patients with PsA, particularly in those with elevated titers of antinuclear antibodies.

Conflict of interests: the authors declare no potential conflict of interests.

\section{REFERENCES}

1. Tasdemir M, Hasan C, Agbas A, et al. Sjögren's syndrome associated with systemic lupus erythematosus. Turk Pediatric Ars. 2016; 51: 166-8.
2. Ciccia F, Triolo G, Rizzo A. Psoriatic arthritis. N Engl J Med. 2017; 376: 2094-5.

3. Cantini F, Piccoli L, Nannini C, et al. Psoriatic arthritis: a systematic review. Int J Rheum Dis. 2010; 13: 300-17.

4. Bell L, Murphy CL, Wynne B, Cunnane G. Acute presentation of arthritis mutilans. J Rheumatol. 2011; 38: 174-5.

5. Alani H, Henty JR, Thompson NL, et al. Systematic review and meta-analysis of the epidemiology of polyautoimmunity in Siögren's syndrome (secondary Sjögren's syndrome) focusing on autoimmune rheumatic diseases. Scand J Rheumatol. 2018; 47: 141-54.

6. Kobak S, Kobak CA, Kabasakal Y, Doganavsargil E. Syögren's syndrome in patients with ankylosing spondylitis. Clin Rheumatol. 2007; 26: $173-5$

7. Whaley K, Chisholm DM, Williamson J, et al. Sjögren's syndrome in psoriatic arthritis, ankylosing spondylitis and Reiter's syndrome. Acta Rheumatol Scand. 1971; 17: 105-14.

8. Rodriguez de la Serna A, Casas Gasso F, Diaz Lopez C, Gieli Ferrer C. Association of Sjögren's syndrome with psoriatic arthritis. Can Med Assoc J. 1984; 131: 1329-32.

9. Collins P, Rogers S, Jackson J, McCartan B. Psoriasis, psoriatic arthritis and possible association with Sjögren's syndrome. Br J Dermatol. 1992; 126: 242-5.

10. Gusis SE, Villa NG, Maldonado Cocco JA, et al. Sjögren syndrome in seronegative spondyloarthropathies: an unusual finding. J Rheumatol. 1994; 21: 771-2.

11. Kemeny-Beke A, Szodoray P. Ocular manifestations of rheumatic diseases. Int Ophthalmol. 2020; 40: 503-10.

12. Scotto di Fazano C, Grilo RM, Vergne P, et al. Is the relationship between spondyloarthropathy and Sjögren's syndrome in women coincidental? A study of 13 cases. Joint Bone Spine. 2002; 69: 383-7.

13. Soscia E, Scarpa R, Cimmino MA, et al. Magnetic resonance imaging of nail unit in psoriatic arthritis. J Rheumatol Suppl. 2009; 83: 42-5.

14. Bruzzese V, Marrese C, Ridola L, Zullo A. Psoriatic arthritis mutilans: case series and literature review. J Rheumatol. 2013; 40: 1233-6.

15. Ferreira MF, Kahem CL, Xavian RM, et al. Treating psoriatic arthritis to target: discordance between physicians and patients' assessment, non adherence, and restricted access to drugs precluded therapy escalation in real-world cohort. Clin Rheumatol. 2019; 38 : 961-8.

16. Husni ME, Merola JF, Davin S. The psycho social burden of psoriatic arthritis. Sem Arthritis Rheum. 2017; 47: 351-60. 